\title{
Cerebellar Liponeurocytoma: Relevant Clinical Cytogenetic Findings
}

\author{
Alexander Tucker · Kritsanapol Boon-Unge ${ }^{1} \cdot$ Nancy McLaughlin $\cdot$ Hassana Ibrahim ${ }^{2}$ \\ Nagesh $\mathrm{Rao}^{3} \cdot$ Neil Martin · Richard Everson · Négar Khanlou ${ }^{1}$ \\ Department of Neurosurgery, 'Division of Neuropathology, Department of Pathology and Laboratory Medicine, ${ }^{2}$ Division of Neuroradiology, Department of Radiological \\ Sciences, ${ }^{3}$ Division of Clinical and Molecular Cytogenetics, Department of Pathology and Laboratory Medicine, University of California-Los Angeles, Los Angeles, CA, USA
}

Cerebellar liponeurocytoma (cLNC) is a glioneuronal tumor first described in 1978. ${ }^{1}$ Histologically, the lesion is similar to medulloblastoma and many pathologists incorrectly describe cLNC as "lipomatous medulloblastoma." Various other nomenclatures have been used to describe cLNC, such as lipomatous glioneurocytoma and lipidized mature neuroectodermal tumor of the cerebellum. ${ }^{2}$ To date, over 40 cases have been reported in the literature, including supratentorial variants. Recent studies have shown that cLNC lesions contain NEUROG1 transcripts (expressed in progenitor cells of the cerebellar ventricular zone) and that they lack ATOH1 or OTX2 transcripts (expressed in progenitor cells of the external granule cell layer). These findings suggest that cLNC cells may originate from GABAergic neurons of the cerebellar ventricular zone.

Ultrastructural analysis of cLNC has demonstrated the presence of lipidized neurocytic cells rather than mature adipocytes. ${ }^{3}$ In 2000, World Health Organization (WHO) changed its classification to include "cerebellar liponeurocytoma," emphasizing the tumor's neurocytic differentiation. Furthermore, in the current classification, CLNC was classified in the category of glioneuronal tumors and upgraded to grade II due to the results of long-term follow-up studies revealing a higher recurrence rate than was originally thought. ${ }^{4}$ For most cases, immunohistochemical and molecular profiling of the tumor is essential in the diagnosis of cLNC. While a typical cytogenetic abnormality is not assigned to cLNC, absence of the molecular hallmarks of

\section{Corresponding Author}

Alexander Tucker, MD

Department of Neurosurgery, University of California-Los Angeles, Suite 562 5th Floor, Wasserman Building, 300 Stein Plaza, Los Angeles, CA 90095-6901, USA

Tel: +1-310-794-7362, Fax: +1-310-267-2707, E-mail: atucker@mednet.ucla.edu

Received: June 10, 2016 Revised: July 20, 2016

Accepted: July 25, 2016 high grade tumors (e.g., $M Y C$ rearrangements as seen in medulloblastoma) are valuable negative findings in the diagnostic work-up. Clinically, pathological diagnosis of cLNC remains challenging with many tumors misdiagnosed for histologic mimics with high-grade potential such as oligodendroglioma and medulloblastoma. In this case report, we describe the clinical, radiographic, and pathological features of a patient with newly diagnosed cLNC. By demonstrating the complete cytogenetic profile of cLNC, we hope to improve recognition and proper diagnosis of this rare entity.

\section{CASE REPORT}

A 41-year-old, right-handed woman without significant past medical or family history presented for evaluation of intermittent, positional headaches for 1 year. These symptoms progressed and became associated with nausea. The patient denied any postural instability, tremor, changes in gait, vision changes, dysphonia, weakness, or sensory disturbance. There was no associated neck or back pain.

Examination at presentation revealed that the patient was awake, alert, and fully oriented. Visual fields were full to confrontation and fundoscopic examination showed no papilledema. The patient had normal facial sensation and facial movement. Tongue and palate were along the midline. Bilateral finger-tonose testing was normal. Strength was full and no pronator drift was detected. Sensation was intact to light touch throughout. No hyperreflexia was noted nor were there signs of lower extremity clonus. An equivocal Babinski sign was noted on the right side, but toes were downgoing on the left. Her gait appeared smooth and Romberg sign was negative.

Magnetic resonance imaging (MRI) of the brain with and 
without contrast was obtained and showed a $4.5 \times 5.2 \times 3.6 \mathrm{~cm}$ (anterior-posterior $\times$ transverse $\times$ cranial-caudal) heterogeneously enhancing mass in the right cerebellar hemisphere. The lesion was hypointense on $\mathrm{T} 1$ and hyperintense on $\mathrm{T} 2$, and it had minimal surrounding edema (Fig. 1A-D). The presence of flow voids on T2 imaging indicated possible high vascularity. It was not entirely clear if the tumor was intra- or extra-axial. Moderate mass effect was evident, resulting in tonsillar herniation of approximately $1.6 \mathrm{~cm}$ into the foramen magnum and compression of the fourth ventricle. Resultant obstructive hydrocephalus was mild, and transependymal transudation was noted on T2 imaging. Complete spine MRI did not show any abnormality.

Suboccipital craniotomy was performed and microsurgical total resection of the tumor was achieved. Grossly, the tumor appeared to be intra-axial with an exophytic component and it was $\mathrm{red} /$ yellow-colored, not hemorrhagic, and well-circumscribed. It originated within the medial aspect of the right cerebellar hemisphere in the region of the vermis underlying the arachnoid (Fig. 1E). It did not involve the dura. Superficially, the tumor had a clearly defined margin with a clean plane between the lesion and the adjacent cerebellar tissue. In the deep aspect of the tumor, however, the interface between the tumor and healthy parenchyma was not as sharply defined.

\section{Histopathology and molecular studies}

Histologic examination of the permanent sections showed a moderately cellular biphasic neoplasm. Areas of tumor cells were characterized by round to elongated nuclei with scant cytoplasm arranged in sheets (Fig. 2A). Foci of oligodendroglia-like tumor cells with central round nuclei and perinuclear halos were arranged in nests, separated by thin branching vessels (Fig. 2B). In other areas, nuclear palisading, perivascular rosetting, and rare Homer-Wright rosettes were seen (Fig. 2C). Nuclear pleomorphism and atypia were minimal. Clusters of lipidized tumor cells reminiscent of mature adipocytes were easily identified within the tumor (Fig. 2D). Mitoses were inconspicuous. Rare microfoci of necrosis were seen whereas pseudopalisading necrosis was absent. Prominent vascular channels with hyalinization and occasional complex vessels were noted within the tumor. Classic microvascular endothelial hyperplasia was not identified.

Immunohistochemistry using antibodies to glial fibrillary acidic protein (GFAP), a glial marker, and synaptophysin, a neuronal marker, were performed. Neoplastic cells showed diffuse and strong immunoreactivity with synaptophysin (Fig. 3A), but failed to react with GFAP. Staining for GFAP and CD34 highlighted background reactive gliosis and blood vessels, respectively (Fig. 3B). Inhibin, CD99, and neurofilament immunoreactions were negative in tumor cells. The proliferative rate based on $\mathrm{Ki}-67$ nuclear labeling index was estimated at 3\%-5\% (Fig. 3C). Although overall morphologic and immunohistochemical profile did not favor a high grade tumor, select molecular studies were performed.

Fluorescent in-situ hybridization (FISH) studies showed no evidence of $1 \mathrm{p} / 19 \mathrm{q}$ co-deletion, $N-M Y C$ or epidermal growth fac-
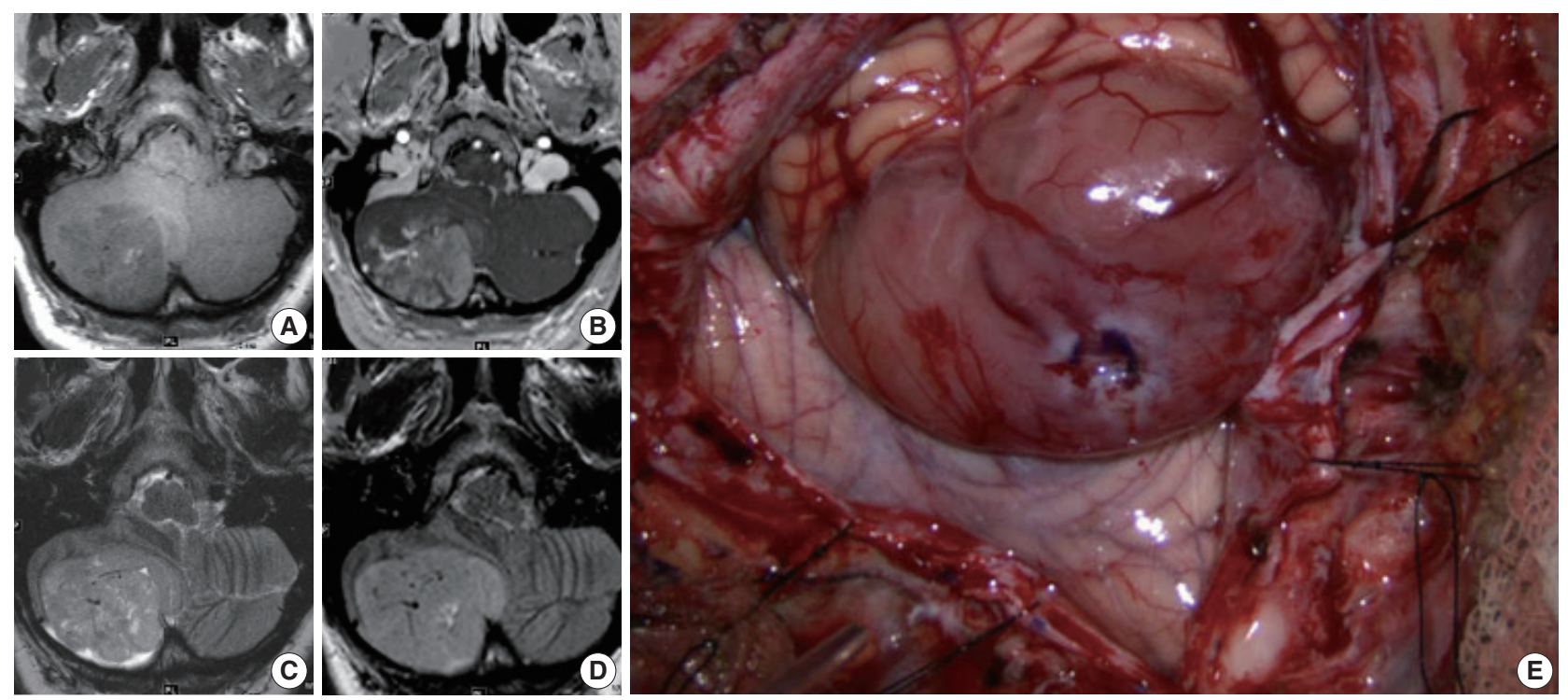

Fig. 1. Radiographic and gross appearance. Magnetic resonance imaging of heterogeneously-enhancing right cerebellar hemisphere mass. Axial T1-weighted precontrast (A), T1-weighted postcontrast (B), T2-weighted (C), and fluid attenuated inversion recovery (D) images through the posterior fossa. Intraoperative photograph of tumor and surrounding cerebellum (E). 
tor receptor $(E G F R)$ amplification, $M Y C$ or EWSR1 rearrangements, or PTEN deletion. However, these studies detected three to six copies each of chromosomes 1, 2, 7, 8q, 10, 19, and 22q in 50\%-80\% ([151-247]/300) of nuclei examined (Fig. 3D).

\section{DISCUSSION}

The case described here highlights the main clinical, radiographic and pathologic features of cLNC. With its histologic similarity to many other neoplasms, surgical pathologists must include CLNC in the differential diagnoses of lesions of the posterior fossa with a clear cell morphology (oligodendroglioma, hemangioblastoma, and clear cell ependymoma), neuroectodermal tumors (medulloblastoma), and tumors with possible lipidized component (glioblastoma and meningioma). Without a clear diagnostic rubric, most histologic and molecular studies aim to exclude tumors of high-grade potential, rendering the diagnosis of cLNC as a diagnosis of exclusion. The importance of accurate diagnosis cannot be overstated, as it used to guide further therapeutic intervention and determination of the patient's prognosis.
Histologically, cLNC appears as monotonous sheets of clear cells or oligodendroglia-like cells, as well as characteristic lipidized cells. Biphasic patterns of dense cellularity alternating with clear cell areas, such as in this case, have also been reported. ${ }^{3}$ Nuclear palisading, true rosette formation and perivascular arrangements were also noted in our case; however, this is not typical. Areas of high cellularity and rosette formation may prompt some pathologists to consider the diagnosis of glioblastoma or ependymoma; however, unlike these entities, cLNC is rarely mitotically active. Features such as necrosis and vascular endothelial hyperplasia, when present, are not correlated with malignant transformation or rapid regrowth and have no effect on overall prognosis. Conversely, there are a few reports describing aggressive clinical tumor behavior in the absence of the classic highgrade features, although this evidence is limited. ${ }^{5}$

The most common alternative diagnosis considered by pathologists reviewing cases of cLNC is medulloblastoma. Medulloblastoma is the most prevalent primary malignancy of the posterior fossa, seen most commonly in children and young adults. In contrast, CLNC is typically diagnosed in adults. Histologically, medulloblastoma has a more primitive or embryonal appear-
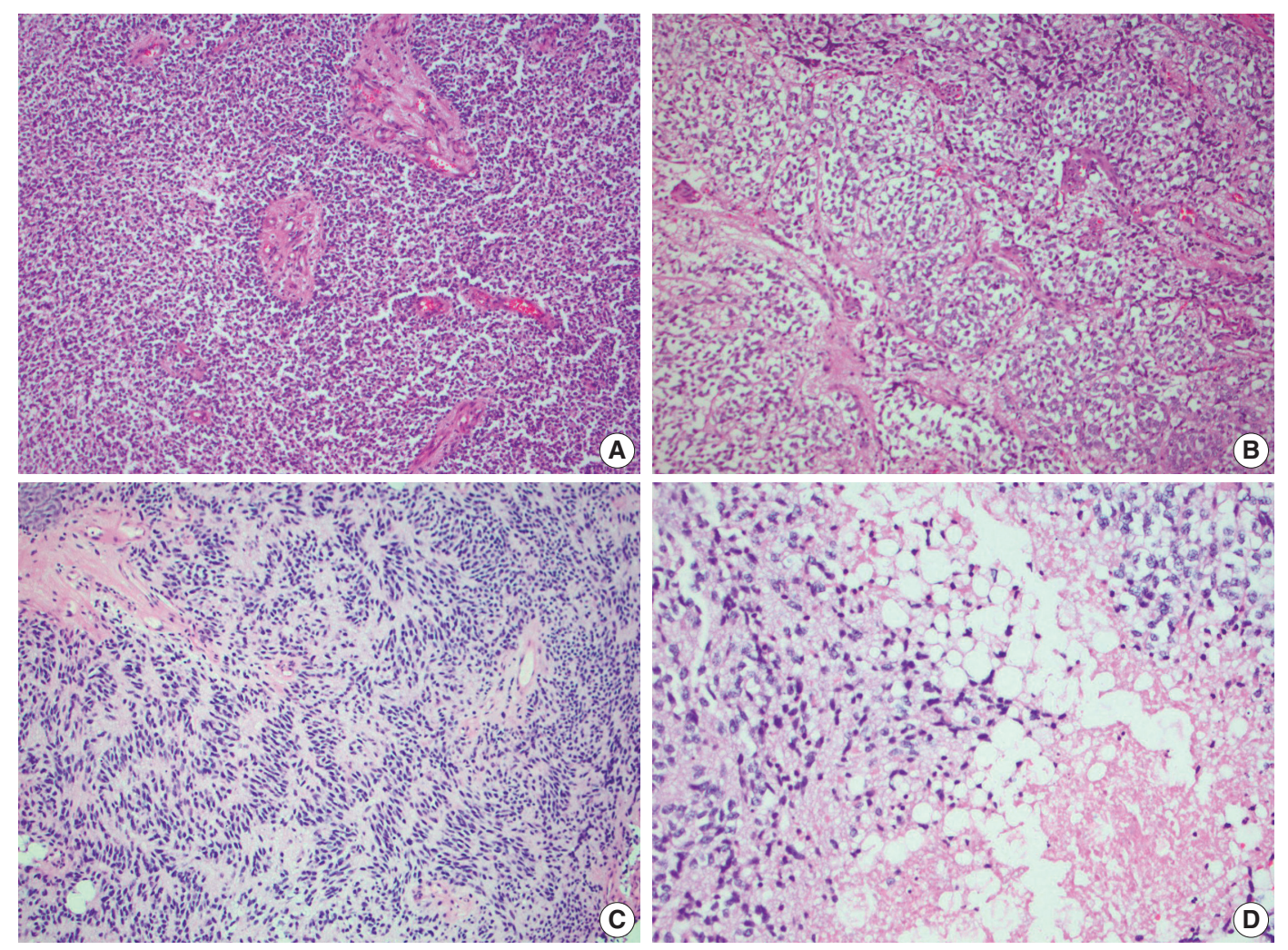

Fig. 2. Hematoxylin and eosin staining. (A) Areas of dense cellularity and scattered prominent vascular channels. (B) Oligodendroglial-like areas demonstrating cells with distinct perinuclear halos and a chicken wire vascular pattern. (C) Region of palisading tumor nuclei reminiscent of ependymoma. (D) Foci of lipidized cells. 
ance than the neurocytic morphology observed in the cLNC. Similarly, anaplasia, nuclear crowding, and high proliferation rates are more common in medulloblastoma than in cLNC. In addition, clear cell morphology and lipidized cells are not typical findings in medulloblastoma, but are seen in cLNC.

Other tumors that are considered part of the differential diagnosis for cLNC include oligodendroglioma and clear cell ependymoma. In general, the diagnosis of oligodendroglioma in the
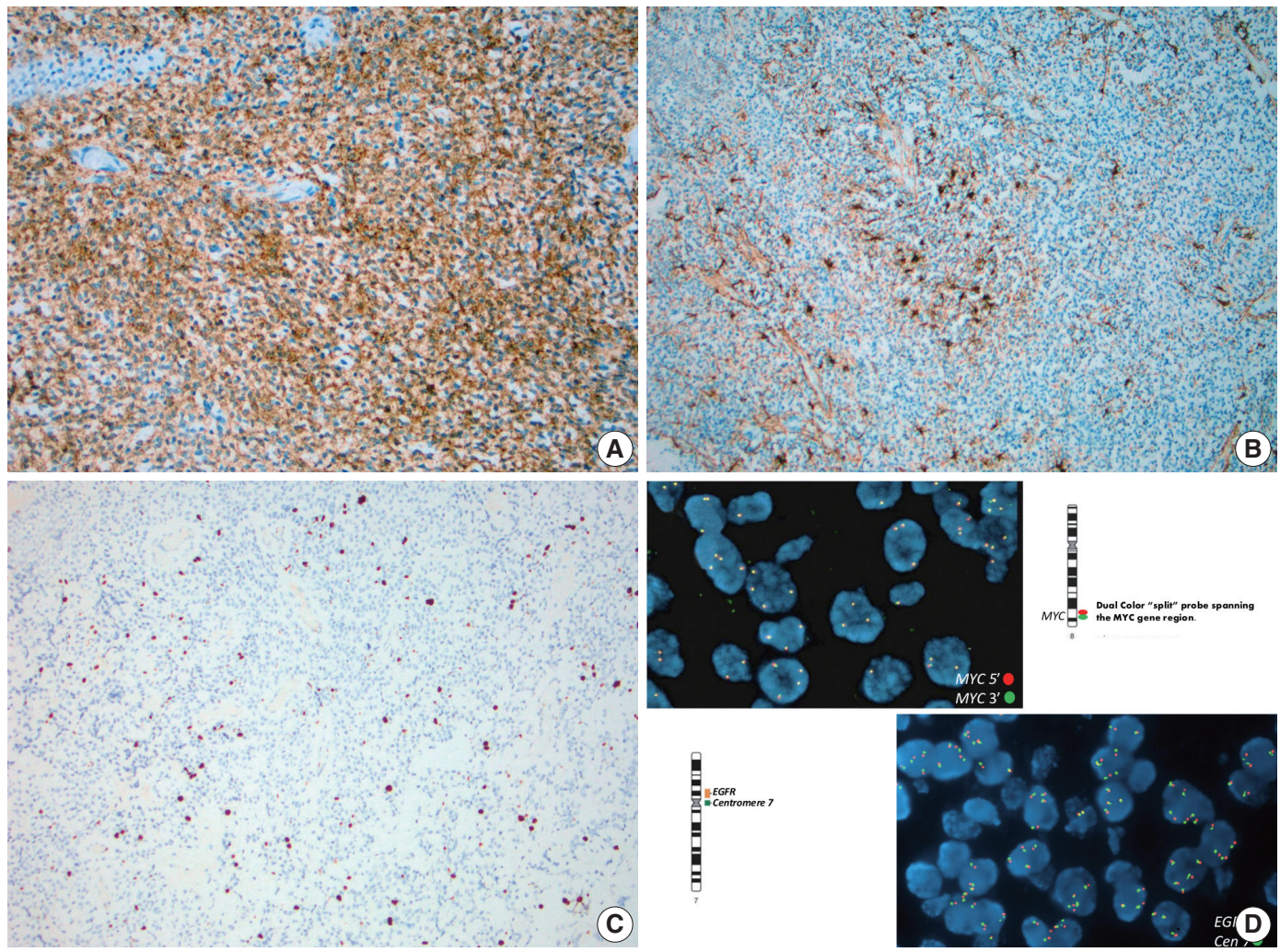

Fig. 3. Immunohistochemistry and fluorescent in-situ hybridization. (A) Diffuse synaptophysin immunoreactivity within the tumor. (B) Reactive gliosis on glial fibrillary acidic protein immunohistochemistry, but no tumor immunoreactivity. (C) Low Ki-67 nuclear labeling index, estimated at 3\%-5\%. (D) Fluorescent in-situ hybridization demonstrating lack of epidermal growth factor receptor (EGFR) amplification or MYC rearrangement.

Table 1. Demographic and pathologic comparison of CLNC and central neurocytoma, clear cell ependymoma, oligodendroglioma, and medulloblastoma

\begin{tabular}{|c|c|c|c|c|c|}
\hline & Cerebellar liponeurocytoma & Central neurocytoma & Clear cell ependymoma & Oligodendroglioma & Medulloblastoma \\
\hline Age of diagnosis (yr) & $50-60$ & $20-40$ & $1-20$ & $30-50$ & $5-30$ \\
\hline WHO grade & $\|$ & $\|$ & $\|$ & II or III & IV \\
\hline Histology & $\begin{array}{l}\text { Neurocytic clear cells } \\
\text { in sheets with lipidized } \\
\text { cells }\end{array}$ & $\begin{array}{l}\text { Clear cells, modest } \\
\text { cytoplasm, salt and } \\
\text { pepper chromatin }\end{array}$ & $\begin{array}{l}\text { Clear cells, round nuclei, } \\
\text { perinuclear halos, } \\
\text { perivascular rosettes }\end{array}$ & $\begin{array}{l}\text { Clear cells, dark nuclei, } \\
\text { "chicken wire" } \\
\text { vasculature }\end{array}$ & $\begin{array}{l}\text { Highly cellular, small, } \\
\text { round, primitive/ } \\
\text { embryonal cells }\end{array}$ \\
\hline High grade features & Uncommon & Uncommon & Common & Common & Common \\
\hline IHC hallmark & $\begin{array}{l}\text { Synaptophysin + } \\
\text { GFAP +/- } \\
\text { Ki-67 <5\% }\end{array}$ & $\begin{array}{l}\text { Synaptophysin + } \\
\text { GFAP - } \\
\text { Ki-67 <5\% }\end{array}$ & $\begin{array}{l}\text { GFAP + } \\
\text { EMA + (dot-like) } \\
\text { Vimentin ++ } \\
\text { Ki-67 <5\% }\end{array}$ & $\begin{array}{l}\text { IDH-1 }(\mathrm{R} 132 \mathrm{H})+/- \\
\text { Olig2 + } \\
\text { Synaptophysin +/- } \\
\text { Ki-67 variable }\end{array}$ & $\begin{array}{l}\text { Synaptophysin ++ } \\
\text { NSE ++ } \\
\text { Vimentin ++ } \\
\text { Ki-67 high (>20\%) }\end{array}$ \\
\hline Genetic hallmark & $\begin{array}{l}\text { Preserved MYC status, } \\
\text { no } 1 \mathrm{p} / 19 \mathrm{q} \text { loss, } \\
\text { no IDH1 mutation, } \\
\text { possible TP53 mutation }\end{array}$ & $\begin{array}{l}\text { Gain } \\
\text { of chromosome } 7\end{array}$ & $\begin{array}{l}\text { Loss } \\
\text { of chromosome } 22\end{array}$ & Dual 1p/19q loss & $\begin{array}{l}\text { Isochromosome } 17 q \text {, } \\
\text { MYC rearrangement }\end{array}$ \\
\hline
\end{tabular}

cLNC, cerebellar liponeurocytoma; WHO, World Health Organization; IHC, immunohistochemisty; GFAP, glial fibrillary acidic protein; EMA, epithelial membrane antigen; IDH-1, isocitrate dehydrogenase 1; NSE, neuron-specific enolase. 
cerebellum should be approached cautiously and cLNC should always be in included in its differential diagnosis. Despite clear cell morphology, oligodendroglioma lacks the extensive neuronal differentiation or lipidization that is found in cLNC. Similarly, clear cell ependymoma is generally strongly immunoreactive to GFAP, lacks lipid-laden cells, and histologically presents with higher-grade nuclear features.

When studied with immunohistochemistry, cLNC is immunoreactive with the most commonly used neuronal markers (i.e., neuron-specific enolase, synaptophysin, Neu-N, chromogranin A, and MAP-2). Glial differentiation and GFAP expression when present are limited. Cases with skeletal muscle differentiation, albeit uncommon, may show desmin expression. ${ }^{6}$ The Ki67 proliferation index for CLNC is generally significantly lower than that for medulloblastoma and other high grade mimics, typically $<5 \%$. In many cases a low mitotic rate and low Ki-67 proliferation index can be used to exclude medulloblastoma as a likely consideration when histological diagnosis is less clear.

Although current literature tends to use histologic and molecular markers for neurocytomas to classify CLNC, recent studies question this practice and suggest that CLNC may have an alternative genetic pathogenesis. In central neurocytoma, polysomic gain of chromosome 7 is found frequently and isolated loss of $1 \mathrm{p}$ and $19 \mathrm{q}$ have also been reported. ${ }^{8}$ In extraventricular neurocytomas, dual 1p/19q loss and particularly t(1;19)(q10; p10), is observed and has been associated with atypical histologic features and increased mitotic activity. ${ }^{9}$ It should be noted that dual $1 \mathrm{p} / 19 \mathrm{q}$ loss is considered the genetic hallmark of oligodendroglioma and is not seen in central neurocytoma. In contrast to central and extraventricular neurocytoma, there are no detailed cytogenetic reports of cLNC tumors.

In the case presented here, there was no evidence of $1 \mathrm{p} / 19 \mathrm{q}$ co-deletion, N-MYC or EGFR amplification, MYC or EWSR1 rearrangements, or PTEN deletion. Cytogenetic studies detected three to six copies each of chromosomes 1, 2, 7, 8q, 10, 19, and 22q. In addition to preserved $M Y C$ status, detailed cluster DNA analyses was used to distinguish cLNC from medulloblastoma. Typically cLNC lacks isochromosome 17 or mutations of the beta-catenin (CTNNB1), PTCH or adenomatous polyposis coli (APC) genes which are often found in medulloblastoma. ${ }^{2,10} \mathrm{Un}$ like astrocytic tumors, $I D H 1$ mutation is not commonly present in CLNC and there was no IDH1 mutation in codon 132 in the case presented here. TP53 mutations are seen in $20 \%$ of the cLNC cases, although not in the case described above, which is a higher rate than in medulloblastoma and usually not found in central neurocytoma. ${ }^{10}$ Recent data show the upregulation of
FABP4, a gene primarily expressed in adipocytes and macrophages. Unfortunately most of these molecular markers are not available to clinical testing. The specificity or prognostic significance of these finding remains to be determined, although it appears likely that CLNC is a genetically distinct entity from other forms of neurocytoma. We recommend that role of FISH studies in the diagnosis of cLNC is to test for clinically relevant genetic abnormalities known to be associated with histologic mimics of cLNC as their absence could be of tremendous diagnostic and prognostic value (Table 1).

According to the WHO classification, CLNC is considered a low-grade lesion; however, long-term follow-up of patients diagnosed with this tumor is imperative. Recent research suggests that the recurrence rate for CLNC may be much higher than previously believed-as high as $40 \%$ within 12 years for patients treated surgically. ${ }^{2}$ Currently, the standard of care for treating these tumors involves surgical resection and serial neuroimaging. Although evidence is limited, some clinicians advocate adjuvant radiotherapy in cases of subtotal resection or recurrence if reoperation is not possible. ${ }^{5}$ Like most primary intracranial neoplasms, extra cranial metastasis is rare and only one case of distant metastases has been reported in the literature. There are currently no accepted histologic features of cLNC that can be relied on to indicate a more aggressive nature of increased likelihood of recurrence. Atypical features including high proliferative index, necrosis, high mitotic activity, and prominent vascular hyperplasia may warrant a close clinical follow-up, although these findings have not yet been correlated with clinical outcome. It remains to be elucidated whether these atypical features are truly predictive of a more malignant biologic behavior since some tumors recur in the absence of atypical histopathology.

This case report is the first complete description of the clinical, radiographic, histologic, immunohistochemical and genetic findings of cLNC. Although no one immunohistochemical marker, genetic profile or histologic feature distinguishes cLNC, a constellation of findings from multiple modalities can be used to correctly diagnosis this lesion. Further study of cLNC will be required to better understand its pathophysiology, identify a unique diagnostic indicator and perhaps find a cure for this rare brain tumor.

\section{Conflicts of Interest}

No potential conflict of interest relevant to this article was reported. 


\section{REFERENCES}

1. Bechtel JT, Patton JM, Takei Y. Mixed mesenchymal and neuroectodermal tumor of the cerebellum. Acta Neuropathol 1978; 41: 261-3.

2. Nishimoto T, Kaya B. Cerebellar liponeurocytoma. Arch Pathol Lab Med 2012; 136: 965-9.

3. George DH, Scheithauer BW. Central liponeurocytoma. Am J Surg Pathol 2001; 25: 1551-5.

4. Louis DN, Ohgaki H, Wiestler OD, et al. The 2007 WHO classification of tumours of the central nervous system. Acta Neuropathol 2007; 114: 97-109.

5. Jenkinson MD, Bosma JJ, Du Plessis D, et al. Cerebellar liponeurocytoma with an unusually aggressive clinical course: case report. Neurosurgery 2003; 53: 1425-7.

6. Gonzalez-Campora R, Weller RO. Lipidized mature neuroectodermal tumour of the cerebellum with myoid differentiation. Neuro- pathol Appl Neurobiol 1998; 24: 397-402.

7. Oudrhiri MY, Raouzi N, El Kacemi I, et al. Understanding cerebellar liponeurocytomas: case report and literature review. Case Rep Neurol Med 2014; 2014: 186826.

8. Taruscio D, Danesi R, Montaldi A, Cerasoli S, Cenacchi G, Giangaspero F. Nonrandom gain of chromosome 7 in central neurocytoma: a chromosomal analysis and fluorescence in situ hybridization study. Virchows Arch 1997; 430: 47-51.

9. Rodriguez FJ, Mota RA, Scheithauer BW, et al. Interphase cytogenetics for $1 \mathrm{p} 19 \mathrm{q}$ and $\mathrm{t}(1 ; 19)(\mathrm{q} 10 ; \mathrm{p} 10)$ may distinguish prognostically relevant subgroups in extraventricular neurocytoma. Brain Pathol 2009; 19: 623-9.

10. Horstmann S, Perry A, Reifenberger G, et al. Genetic and expression profiles of cerebellar liponeurocytomas. Brain Pathol 2004; 14: 281-9. 